\title{
A Double-Distribution Statistical Algorithm for Composite Laminate Optimization
}

\author{
Laurent Grosset* and Rodolphe Le Riche ${ }^{\dagger}$ \\ École des Mines, Saint-Étienne, France \\ Raphael T. Haftka $a^{\ddagger}$ \\ University of Florida, Gainesville, USA
}

\begin{abstract}
The paper proposes a new evolutionary algorithm for composite laminate optimization, named Double-Distribution Optimization Algorithm (DDOA). The DDOA belongs to the family of Estimation of Distributions Algorithms (EDA) that build a statistical model of promising regions of the design space based on sets of good points, and use it to guide the search. A generic framework for introducing variable dependencies by making use of the physics of the problem is presented. The algorithm uses two distributions simultaneously: a simple distribution for the design variables, complemented by the distribution of auxiliary variables. The combination of the two generates complex distributions at a low computational cost. The paper demonstrates DDOA's efficiency for two laminate optimization problems for which the design variables are the fiber angles and the auxiliary variables are the lamination parameters. The results show that its reliability in finding the optima is greater than that of a simple EDA and of a standard GA, and that its superiority increases with the problem dimension.
\end{abstract}

\section{Introduction}

Stacking sequence optimization of composite laminates is concerned with finding the optimal orientation of each ply. Because of availability of data on failure modes, fiber angles are usually restricted to a finite set of values. This constraint gives rise to combinatorial optimization problems. In the last decade, evolutionary algorithms (EA) have been used to address stacking sequence optimization problems. ${ }^{1-3}$ Standard EAs are population-based algorithms that search the design space by combining portions of high quality solutions (crossover) and mixing it with local random exploration (mutation). While these algorithms have been used with success to solve a wide variety of problems, they typically require the user to tune many parameters by trial and error to maximize efficiency. A second limitation of standard EAs is the lack of theoretical support for their performance.

To address these shortcomings, a new class of evolutionary algorithms called Estimation of Distribution Algorithms (EDAs) has been proposed in the last few years. ${ }^{4}$ EDAs partly abandon the analogy to the Darwinian theory of evolution. Instead, they use a statistical framework to formalize the search mechanisms of EAs. This approach produces algorithms whose behavior is determined by statistically meaningful parameters. The present paper introduces an estimation of distribution algorithm for laminate optimization. The algorithm, called Double-Distribution Optimization Algorithm (DDOA), takes advantage of the physics of the problem to maximize efficiency: it combines statistical information on the fiber angles and auxiliary variables called the lamination parameters to guide the search.

The paper is organized as follows: Section II introduces the principles of estimation of distribution algorithms, Section III gives a review of lamination parameters, Section IV presents the Double-Distribution

\footnotetext{
${ }^{*}$ Graduate Student, SMS/MEM, École des Mines de Saint-Étienne, 42023 Saint-Étienne cedex 02, France.

†Permanent Researcher, CNRS UMR 5146 / SMS, École des Mines de Saint-Étienne, 42023 Saint-Étienne cedex 02, France.

${ }^{\ddagger}$ Distinguished Professor, Department of Mechanical and Aerospace Engineering, Gainesville, FL 32611-6250, USA, AIAA Fellow.
} 
Optimization Algorithm, Section V demonstrates DDOA's efficiency on an application to laminate optimization problems, possible improvements are discussed in Section VI, and Section VII provides conclusions and perspectives.

\section{Estimation of Distribution Algorithms}

Let us consider the optimization problem of maximizing the fitness function $F(\boldsymbol{\theta})$ with $\boldsymbol{\theta}=\left(\theta_{1}, \theta_{2}, \ldots, \theta_{n}\right)$, such that $\theta_{k} \in \mathbb{A}$, a discrete alphabet. Evolutionary algorithms search the design space by iteratively creating populations of points by recombining information from good individuals of the previous generation. In standard EAs, a new population is created by applying crossover and mutation operators to a subset of individuals obtained by applying a fitness-based selection procedure to the population. In estimation of distribution algorithms, the subset of selected points is viewed as a sample from a certain distribution $p^{t}\left(\theta_{1}, \ldots, \theta_{n}\right)$, the distribution of all good points at generation $t$. The set of all good points at generation $t$ is determined by: (1) the distribution of the points before selection and (2) the selection procedure adopted. By iteratively sampling points from $p^{t}\left(\theta_{1}, \ldots, \theta_{n}\right)$, applying a selection operator and explicitly estimating $p^{t+1}\left(\theta_{1}, \ldots, \theta_{n}\right)$, the algorithm visits increasingly fit regions of the search space. The general EDA algorithm is the following:

1. initialize $t \leftarrow 0$ and $p^{0}\left(\theta_{1}, \ldots, \theta_{n}\right)$;

2. if $t \leq t_{\max }$, create a population of $\lambda$ individuals according to $p^{t}\left(\theta_{1}, \ldots, \theta_{n}\right)$

3. evaluate the fitness $F\left(\theta_{1}, \ldots, \theta_{n}\right)$ of the $\lambda$ individuals;

4. select $\mu$ "good points" among the $\lambda$ individuals;

5. estimate the distribution $p^{t+1}\left(\theta_{1}, \ldots, \theta_{n}\right)$ of the $\mu$ selected individuals;

6 . increment $t$ and go to 2 .

In practice, the distribution of promising points $p^{t}\left(\theta_{1}, \ldots, \theta_{n}\right)$ has to be estimated from a finite number $\mu$ of selected points and it is replaced by its approximation $\widehat{p}^{t}\left(\theta_{1}, \ldots, \theta_{n}\right)$. Obtaining a good estimate of that distribution is critical for the algorithm's efficiency, as erroneous probabilities result in wasteful function evaluations. Clearly, a trade-off has to be made between the model flexibility (its ability to represent complex variable interactions) and its stability to the choice of selected individuals (confidence on the model parameters).

Early works assumed simple probability structures. For example, the Probability-Based Incremental Learning algorithm ${ }^{5}$ and the Univariate Marginal Distribution Algorithm ${ }^{6}$ (UMDA) neglected all variable interactions. While this approach has been successful for problems with mild variable interactions, univariate distributions cannot capture dependencies between variables and can drive the optimization to a local optimum. Mühlenbein and Mahnig ${ }^{7}$ give an example of a deceptive problem where univariate distributions are misleading. The presence of constraints can introduce variable dependencies in the population of selected points, as illustrated by the 2D example shown in Figure 1. After selection, the points follow the contours of the penalized fitness function. The probability distribution obtained by neglecting variable interactions favors regions that are far from the optimum. For problems with strong variable interactions, one needs to use more complex distributions. Several researchers have proposed EDAs with higher-order distributions. In the MIMIC algorithm, ${ }^{8}$ pairwise interactions between variables is incorporated by through a chain model.

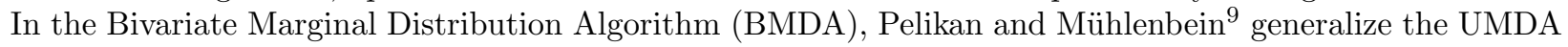
algorithm by using a tree model. Even higher-order models have been proposed: the Factorized Distribution Algorithm ${ }^{10}$ uses the structure of the fitness function to simplify the distribution representation. The Bayesian Optimization Algorithm, proposed by Pelikan et al., ${ }^{11}$ represents the distribution of good selected individuals by Bayesian networks, thereby allowing any degree of complexity for the distribution. In the present paper, we propose a physics-based strategy for incorporating variable dependencies into the probabilistic model. 


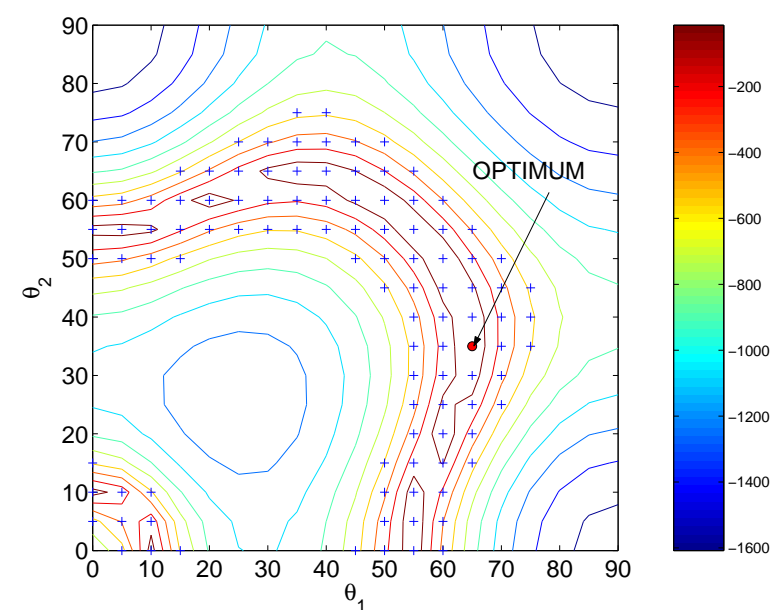

(a) Fitness landscape and selected points.

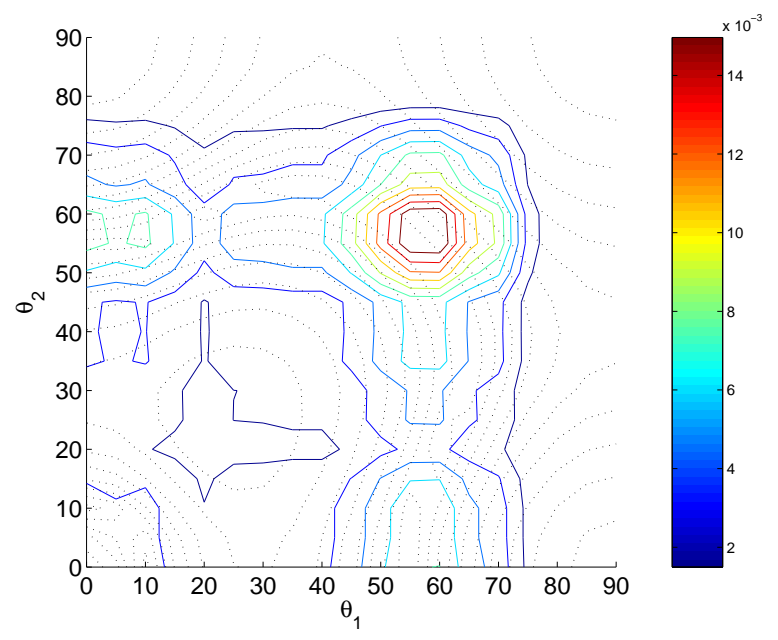

(b) Univariate distribution.

Figure 1. Contours of the univariate distribution (b) based on the selected points (a). The high probability areas do not coincide with high fitness regions.

\section{Lamination Parameters}

In laminate stacking sequence optimization, the overall stiffness properties of a laminate are completely captured by the "lamination parameters". ${ }^{12}$ The utility of lamination parameters for composite optimization has been demonstrated by several researchers. ${ }^{13,14}$ Performing the optimization in the lamination parameter space has several advantages: in some cases, it forces convexity of the fitness function, ${ }^{15}$ it reduces dimensionality, it renders the problem amenable to continuous optimization methods.

The only non-zero extensional lamination parameters $V_{i}^{*}$ and flexural lamination parameters $W_{i}^{*}$ of a balanced symmetric laminate $\left[ \pm \theta_{1}, \pm \theta_{2}, \cdots, \pm \theta_{n}\right]_{s}$ are, respectively, the following:

$$
\begin{aligned}
V_{\{1,3\}}^{*} & =\frac{2}{h} \int_{0}^{h / 2}\{\cos 2 \theta, \cos 4 \theta\} d z \\
& =\frac{1}{n} \sum_{k=1}^{n}\left\{\cos 2 \theta_{k}, \cos 4 \theta_{k}\right\}
\end{aligned}
$$

and

$$
\begin{aligned}
W_{\{1,3\}}^{*} & =\frac{24}{h^{3}} \int_{0}^{h / 2}\left\{\cos 2 \theta_{k}, \cos 4 \theta_{k}\right\} z^{2} d z \\
& =\frac{1}{n^{3}} \sum_{k=1}^{n} a_{k}\left\{\cos 2 \theta_{k}, \cos 4 \theta_{k}\right\}
\end{aligned}
$$

where $h$ designates the total laminate thickness and $a_{k}=(n-k+1)^{3}-(n-k)^{3}$. The ply numbering convention adopted here is shown in Figure 2.

The stiffness matrix components of a balanced symmetric laminate can be expressed as linear functions of the lamination parameters as follows: ${ }^{16}$

$$
\left\{\begin{array}{l}
A_{11} \\
A_{22} \\
A_{12} \\
A_{66}
\end{array}\right\}=h\left[\begin{array}{ccc}
U_{1} & V_{1}^{*} & V_{3}^{*} \\
U_{1} & -V_{1}^{*} & V_{3}^{*} \\
U_{4} & 0 & -V_{3}^{*} \\
U_{5} & 0 & -V_{3}^{*}
\end{array}\right]\left\{\begin{array}{c}
1 \\
U_{2} \\
U_{3}
\end{array}\right\}
$$




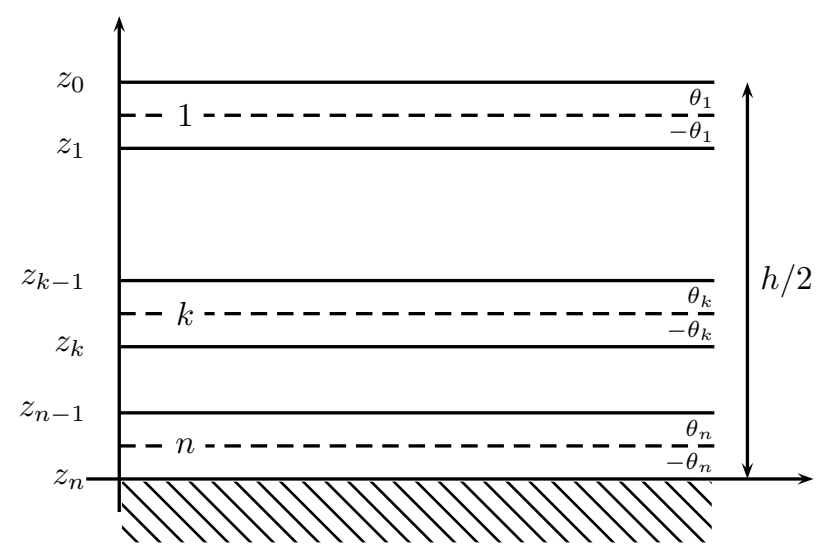

Figure 2. Ply-numbering convention for a balanced symmetric laminate.

and

$$
\left\{\begin{array}{c}
D_{11} \\
D_{22} \\
D_{12} \\
D_{66}
\end{array}\right\}=\frac{h^{3}}{12}\left[\begin{array}{ccc}
U_{1} & W_{1}^{*} & W_{3}^{*} \\
U_{1} & -W_{1}^{*} & W_{3}^{*} \\
U_{4} & 0 & -W_{3}^{*} \\
U_{5} & 0 & -W_{3}^{*}
\end{array}\right]\left\{\begin{array}{c}
1 \\
U_{2} \\
U_{3}
\end{array}\right\},
$$

where $U_{1}, U_{2}, U_{3}, U_{4}$, and $U_{5}$ are the material invariants of the laminate.

\section{The Double-Distribution Optimization Algorithm}

In the present paper, we propose a physics-based strategy for incorporating variable dependencies: instead of using a complex model to represent the interactions between the $\theta_{k}$ 's, we observe that variable dependencies among selected points often reflect the fact that the overall response of the system is really a function of integral quantities, so that many combinations of the design variables can produce the same response. For example, the dimensions of the section of a beam determine its flexural behavior through the moment of inertia, the aerodynamic properties of a vehicle is captured by the drag coefficient, etc. These quantities are typically inexpensive to calculate, and their number is limited and insensitive to the number of design variables. In the case of laminates, the lamination parameters are such quantities.

The proposed algorithm, named Double-Distribution Optimization Algorithm (DDOA) uses a simple univariate model to represent the angle distribution $p\left(\theta_{1}, \ldots, \theta_{n}\right)$, but introduces variable dependencies by biasing the search based on the continuous distribution of selected individuals in the lamination parameter space $^{\mathrm{a}}$. Let $f(\mathbf{V})$ designate that distribution ( $\mathbf{V}$ is the set of lamination parameters considered and depends on the problem at hand: $\mathbf{V}=\left(V_{1}^{*}, V_{3}^{*}\right)$ for in-plane problems, $\mathbf{V}=\left(W_{1}^{*}, W_{3}^{*}\right)$ for out-of-plane problems, and $\mathbf{V}=\left(V_{1}^{*}, V_{3}^{*}, W_{1}^{*}, W_{3}^{*}\right)$ for problems involving both the extensional and flexural properties of the laminate). The DDOA algorithm is presented in Figure 3.

The two search distributions cooperate in the following manner: in problems that can be expressed as functions of the lamination parameters only, the distribution $f(\mathbf{V})$ accurately describes the promising regions. It can therefore be regarded as an "ideal" search distribution. To approximate that desirable distribution in the $\mathbf{V}$-space, while generating points in the $\theta$-space, a three-step strategy is proposed, where the angle distribution provides a set of points that have correct univariate distributions, while the lamination parameter distribution introduces variable dependencies. First, a set a set of $\lambda$ points is created in the Vspace by sampling from $f(\mathbf{V})$. Then, a larger set of $\nu$ candidate points is sampled from $p\left(\theta_{1}, \ldots, \theta_{n}\right)$. Finally, the $\lambda$ candidate points that are closest to the target points in the $\mathbf{V}$-space are accepted as the new population (Euclidean distances between all possible target-candidate pairs are calculated, then for each target point, the closest candidate point is accepted for the next generation. Any pair that has been used is then disregarded for the rest of the acceptance procedure). Depending on the degree of confidence placed in the distributions

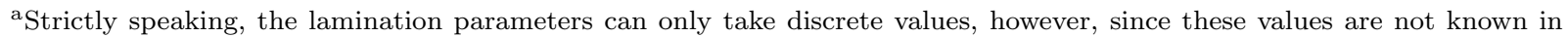
advance, their distribution is best described by a continuous model.
} 


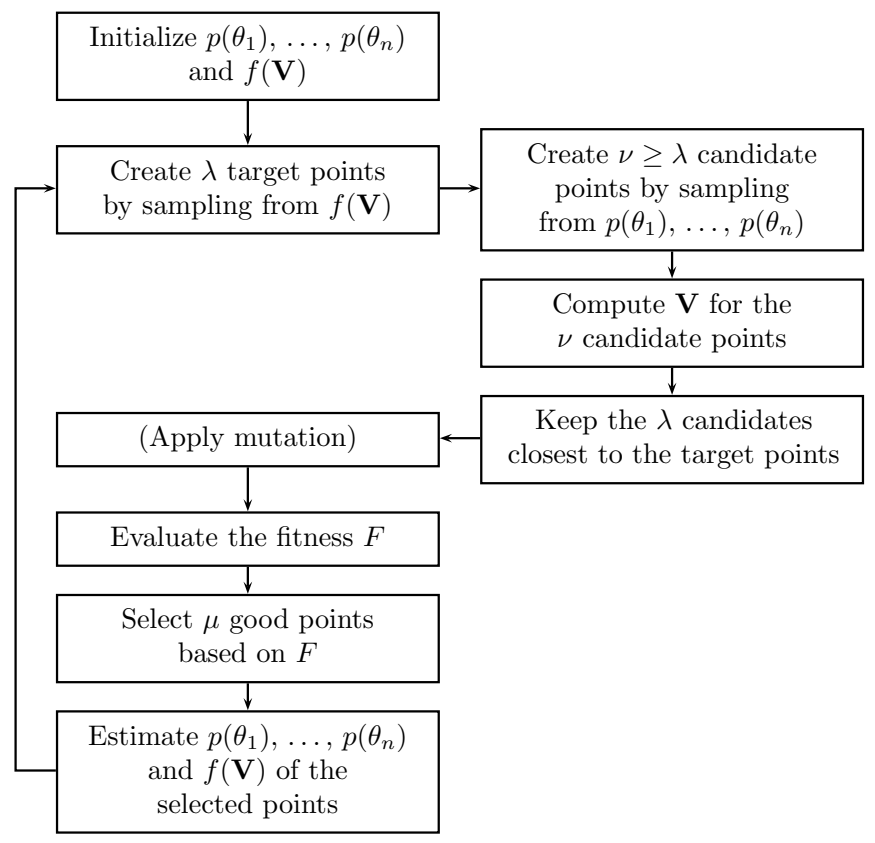

Figure 3. The DDOA algorithm

$p\left(\theta_{1}, \ldots, \theta_{n}\right)$ and $f(\mathbf{V})$, their relative influence in the creation of new points can be adjusted through the ratio $\nu / \lambda$ : when $\nu / \lambda=1$, the lamination parameter distribution $f(\mathbf{V})$ plays no role in the search; when $\nu / \lambda \rightarrow \infty$, the optimization is based primarily on information in the lamination parameter space. In our implementation, we provided the possibility of applying mutation to allow lost values to be reintroduced in the population. Fitness-based selection is then applied to the population, and the distributions $p\left(\theta_{k}\right)$, $k=1, \ldots, n$ and $f(\mathbf{V})$ of the selected points are estimated.

Two aspects of the algorithm deserve a more detailed description: the representation and the estimation of $f(\mathbf{V})$ and the creation procedure of target points in the $\mathbf{V}$-space. Most of the works on continuous EDAs to date use univariate normal distributions to represent the distribution of selected points. ${ }^{17,18}$ The disadvantages of that model is that it does not model dependencies between variables, and assumes a unimodal symmetric distribution. Bosnan and Thierens ${ }^{19}$ propose to use a non-parametric kernel density estimation (KDE) method to achieve a more accurate approximation. Since the number of lamination parameters that we consider is small (two to four) and independent of the problem dimension, KDE is appropriate for modeling the distribution $f(\mathbf{V})$. In the kernel density estimation method, a kernel $K(\mathbf{u})$ is placed at each sample point. The distribution $f(\mathbf{V})$ is obtained as

$$
f(\mathbf{V})=\frac{1}{\mu} \sum_{i=1}^{\mu} K\left(\mathbf{V}-\mathbf{V}_{i}\right) .
$$

In this work, we used Gaussian kernels:

$$
K(\mathbf{u})=\frac{1}{(2 \pi)^{d / 2} \sigma^{d}} \exp \left(\frac{\mathbf{u}^{T} \mathbf{u}}{\sigma^{2}}\right)
$$

where $d$ is the dimension of $\mathbf{u}$ and the variance $\sigma^{2}$ is the bandwidth that needs to be adjusted: a small value of $\sigma$ increases the resolution but also increases the variance of the estimate when few data points are available. Several methods for adjusting the value of $\sigma$ exist: trial-and-error, maximum likelihood, or adaptive strategy. In this work, we adopted a maximum likelihood method, presented in Appendix.

The use of unbounded-support density functions, such as normal kernels, for the representation of $f(\mathbf{V})$ complicates the sampling of points in the bounded lamination parameter space, because infeasible target points can be generated. Two strategies were considered: one can either force the target points to lie in the feasible domain by sampling new points until feasible points are obtained, or allow infeasible target points 
to be created, considering that all the points of the new generation will be feasible by construction. The former approach has two disadvantages: first, the exact boundaries of the feasible domain are only known for a few simple combinations of lamination parameters $\left(\left(V_{1}^{*}, V_{3}^{*}\right)\right.$ and $\left.\left(V_{1}^{*}, V_{3}^{*}\right)\right)$. For general combinations, approximate relations only are available. ${ }^{20}$ Second, tests showed that the performance of algorithms based on the rejection of infeasible target points deteriorates when the optima lie close to the boundary. Consequently, the latter scheme only is presented in the present paper.

\section{Application to Laminate Optimization}

We applied DDOA to two laminate optimization problems: an in-plane problem, where the fitness function can be expressed in terms of $V_{1}^{*}$ and $V_{3}^{*}$ only, and a problem involving both extensional and flexural properties, where the fitness is a function of the four lamination parameters $V_{1}^{*}, V_{3}^{*}, W_{1}^{*}$ and $W_{3}^{*}$.

In-Plane PRoBlem. The first problem was maximizing the transverse in-plane stiffness coefficient $A_{22}$ subject to a constraint on the effective Poisson's ratio $\nu_{\text {eff }}$ :

$$
\begin{aligned}
& \text { maximize } A_{22}=h\left(U_{1}-U_{2} V_{1}^{*}+U_{3} V_{3}^{*}\right) \\
& \text { such that } \nu_{1} \leq \nu_{\mathrm{eff}} \leq \nu_{\mathrm{u}}
\end{aligned}
$$

The effective Poisson's ratio $\nu_{\text {eff }}$ is a function of $V_{1}^{*}$ and $V_{3}^{*}$ :

$$
\nu_{\mathrm{eff}}=\frac{A_{12}}{A_{22}}=\frac{U_{4}-U_{3} V_{3}^{*}}{1-U_{2} V_{1}^{*}+U_{3} V_{3}^{*}},
$$

and $U_{1}, U_{2}, U_{3}, U_{4}$ are material invariants, obtained from the material properties shown in Table 1 , and the total laminate thickness was fixed at $h=0.2 \mathrm{in}^{\mathrm{b}}$. The lower and upper limits of the Poisson's ratio were $\nu_{1}=0.48$ and $\nu_{\mathrm{u}}=0.52$. The inequality constraints were enforced by a penalty approach:

$$
F(\boldsymbol{\theta})= \begin{cases}A_{22} & \text { if } g(\boldsymbol{\theta}) \geq 0 \\ A_{22}+p g(\boldsymbol{\theta}) & \text { if } g(\boldsymbol{\theta})<0\end{cases}
$$

where

$$
g(\boldsymbol{\theta})=\min \left(\frac{\nu_{\mathrm{eff}}}{\nu_{1}}-1,1-\frac{\nu_{\mathrm{eff}}}{\nu_{\mathrm{u}}}\right)
$$

The value of the penalty parameter $p$ was set to $5.010^{6}$ so as to guaranty a feasible solution. The fiber orientations were chosen from $\left\{0^{\circ}, 22.5^{\circ}, 45^{\circ}, 67.5^{\circ}, 90^{\circ}\right\}$. This problem is an ideal case for the DDOA algorithm, because the objective function can be expressed entirely in terms of $V_{1}^{*}$ and $V_{3}^{*}$, so that the distribution of promising points can be characterized completely in the $\left(V_{1}^{*}, V_{3}^{*}\right)$-plane. In this problem, variable interactions originate from two factors. First, the constraint creates a "tunnel" in the design space and gives rise to variable dependencies, as in the previous example. Second, since the order of the plies is irrelevant, several configurations of the optimal laminate may be present in the set of selected points, hence creating additional variable dependencies.

Two cases were tested: $n=6$ and $n=12$. In the former case, the optimum stacking sequence was $\left[ \pm 45_{4} / \pm 67.5_{2}\right]_{s}$ (or any permutation of the same angles), with a transverse stiffness $A_{22}=1.89710^{6} \mathrm{lb} / \mathrm{in}$, and an effective Poisson's ratio $\nu_{\text {eff }}=0.519$. In the case $n=12$, the optimum stacking sequence was $\left[ \pm 45_{9} / \pm 67.5 / 90_{4}\right]_{s}$ (or any permutation of the same angles), with a transverse stiffness $A_{22}=1.92210^{6}$ $\mathrm{lb} / \mathrm{in}$, and an effective Poisson's ratio $\nu_{\text {eff }}=0.489^{\mathrm{c}}$.

The DDOA algorithm was compared to two evolutionary algorithms: a standard genetic algorithm (population size of 30 , two-point crossover with probability $p_{c}=1.0$, linear ranking parent selection) and the univariate marginal distribution algorithm ${ }^{6}$ with linear ranking selection, population size $\lambda=30$ and selected population size $\mu=30$. These settings were also used for DDOA, and a candidate pool size $\nu=150$

\footnotetext{
${ }^{\mathrm{b}}$ In practice, the ply thickness is fixed, and the total thickness depends on the number of plies. However, we used a fixed total thickness in this work to observe the effect of dimensionality for a given fitness function.

${ }^{\mathrm{c}}$ The constraint is not strictly active because of the discreteness of the variables.
} 
Table 1. Material properties of graphite-epoxy.

\begin{tabular}{lr}
\hline Longitudinal modulus, $E_{1}$ & $2.1810^{7} \mathrm{psi}$ \\
Transverse modulus, $E_{2}$ & $1.3810^{6} \mathrm{psi}$ \\
Shear modulus, $G_{12}$ & $1.5510^{6} \mathrm{psi}$ \\
Poisson's ratio, $\nu_{12}$ & 0.26 \\
Material invariant $U_{1}$ & $0.889710^{7} \mathrm{psi}$ \\
Material invariant $U_{2}$ & $1.025410^{7} \mathrm{psi}$ \\
Material invariant $U_{3}$ & $0.274210^{7} \mathrm{psi}$ \\
Material invariant $U_{4}$ & $0.310310^{7} \mathrm{psi}$ \\
Material invariant $U_{5}$ & $0.289710^{7} \mathrm{psi}$ \\
\hline
\end{tabular}

was chosen. In the three algorithms, mutation consisted in changing the value of a gene to one of the two nearest values. A fixed mutation rate $p_{m}=0.02$ per gene was used. The same coding was used for the three algorithms. No elitist strategy was implemented, to isolate the effect of the probabilistic model ${ }^{\mathrm{d}}$. The bandwidth was chosen empirically by a maximum likelihood method, which provides an upper bound for the optimal bandwidth by determining the value of $\sigma$ that best approximates the distribution of selected points at the first generation of the algorithm (see details in Appendix). Using this approach we obtained $\sigma=0.2$ for $n=6$ and $\sigma=0.15$ for $n=12$.

The performance of the optimization was assessed by estimating its reliability $R$, defined as the probability of finding the (practical) optimum at a certain number of function evaluations. The reliability was estimated as the proportion of runs that found the optimum, over 50 independent runs, so that its standard error was $\sigma_{\mathbf{e}}=\frac{\sqrt{R(1-R)}}{\sqrt{50}}$, which is maximum at 0.07 for $R=0.5$. In the case $n=12$, the convergence criterion was relaxed by using a "practical" optimum, defined as $99 \%$ of the maximum fitness, instead of the true optimum. In all cases, the cumulative reliability (reliability of the algorithm when memory is used) is plotted in dashed line. The reliability of the three algorithms is compared in Figure 4. UMDA clearly benefited from the input of information about the lamination parameter distribution. The superiority of DDOA over the GA and UMDA is visible in both cases. The performance improvement can be attributed to a more accurate estimation of the distribution of selected points achieved by incorporating variable dependencies via the lamination parameters. Working in the $\left(V_{1}^{*}, V_{3}^{*}\right)$-plane was especially beneficial for this problem where the ply order is irrelevant because all the configurations of a given laminate get mapped into a single point, so that the distribution becomes very simple in the $\left(V_{1}^{*}, V_{3}^{*}\right)$-plane, while a univariate model in the $\boldsymbol{\theta}$-space describes the distribution only imperfectly. The comparison of the two figures indicates that the gain increases with dimensionality. This can be explain by the fact that, DDOA performs part of the data analysis in the $\left(V_{1}^{*}, V_{3}^{*}\right)$-plane, whose dimension is invariant. Consequently, using the lamination parameters to guide the search partially neutralizes the effect of dimensionality on the reliability.

Flexural/extensional problem. The second problem was minimizing the absolute value of the longitudinal coefficient of thermal expansion (CTE) $\bar{\alpha}_{x}$ of a simply supported rectangular graphite-epoxy plate subject to a lower bound on the first natural frequency $f_{1}$. The problem was formulated as follows:

$$
\begin{aligned}
& \operatorname{minimize}\left|\bar{\alpha}_{x}\right| \\
& \text { such that } f_{1} \geq f_{\min }
\end{aligned}
$$

where the longitudinal CTE is a function of $V_{1}^{*}$ and $V_{3}^{*}: 16$

$$
\bar{\alpha}_{x}=\frac{V_{1}^{*}\left(K_{2} U_{1}-K_{1} U_{2}+K_{2} U_{4}\right)-V_{1}^{* 2} K_{2} U_{2}+2 K_{1} U_{3} V_{3}^{*}+K_{1}\left(U_{1}-U_{4}\right)}{2\left(2 V_{3}^{*} U_{3}\left(U_{4}+U_{1}\right)-V_{1}^{* 2} U_{2}^{2}+\left(U_{1}^{2}-U_{4}^{2}\right)\right)}
$$

\footnotetext{
${ }^{\mathrm{d}}$ However the best solution found at each generation is recorded, so that even though it does not influence the search, it is not lost for the user.
} 


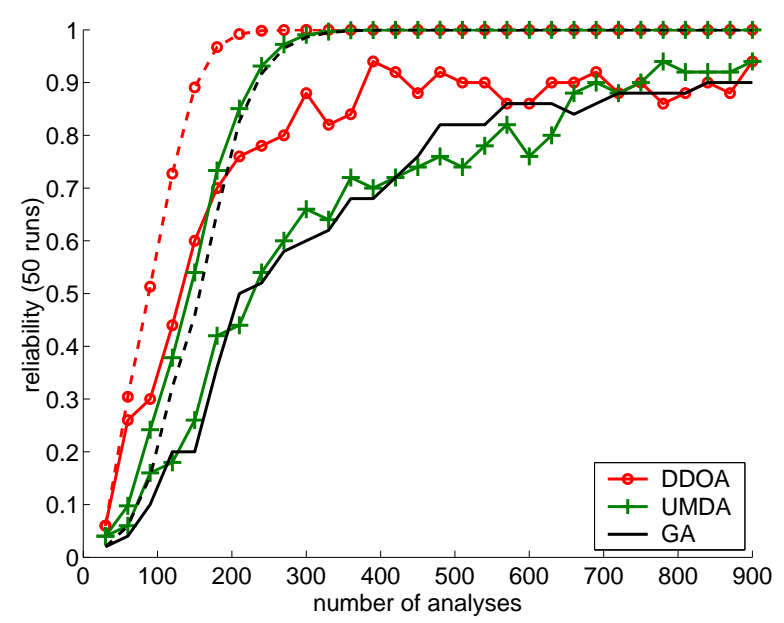

(a)

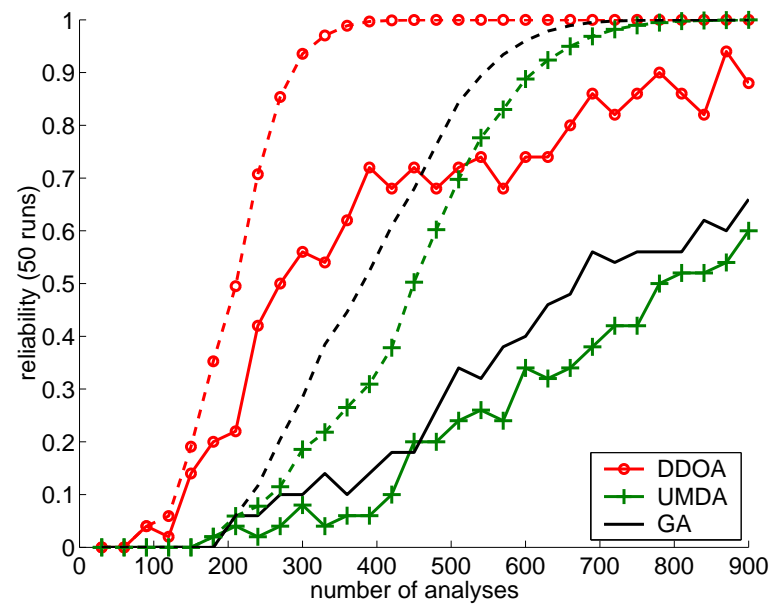

(b)

Figure 4. Reliability (solid line) and cumulative reliability (reliability of the algorithm when the best points visited are stored, dashed line) of a standard genetic algorithm, the univariate marginal distribution algorithm, and the double-distribution algorithm for the in-plane laminate optimization problem (6 variables (a) and 12 variables (b)).

where

$$
\begin{aligned}
& K_{1}=\left(U_{1}+U_{4}\right)\left(\alpha_{1}+\alpha_{2}\right)+U_{2}\left(\alpha_{1}-\alpha_{2}\right) \\
& K_{2}=U_{2}\left(\alpha_{1}+\alpha_{2}\right)+\left(U_{1}+2 U_{3}-U_{4}\right)\left(\alpha_{1}-\alpha_{2}\right) \\
& K_{3}=U_{2}\left(\alpha_{1}+\alpha_{2}\right)+2\left(U_{3}+U_{5}\right)\left(\alpha_{1}-\alpha_{2}\right) .
\end{aligned}
$$

The first natural frequency is a function of $W_{1}^{*}$ and $W_{3}^{*}$ :

$$
f_{1}=\frac{\pi^{2}}{\sqrt{\rho h}} \sqrt{\frac{1}{a^{4}} D_{11}+\frac{2}{a^{2} b^{2}}\left(D_{12}+2 D_{66}\right)+\frac{1}{b^{4}} D_{22}} .
$$

Note that the fitness function can be expressed in terms of the lamination parameters only, as in the previous problem.

For this problem, the material properties shown in Table 1 were used. The coefficients of thermal expansion in the longitudinal and transverse directions were $\alpha_{1}=0.0210^{-6} \mathrm{~K}^{-1}$ and $\alpha_{2}=22.5010^{-6} \mathrm{~K}^{-1}$, respectively, and the dimensions of the plate were $a=30$ in and $b=15 \mathrm{in}$. The minimum frequency was $f_{\min }=150 \mathrm{~Hz}$.

The minimization problem was recast into a maximization problem by defining the fitness function as follows:

$$
F(\boldsymbol{\theta})= \begin{cases}\alpha_{2}-\left|\bar{\alpha}_{x}\right| & \text { if } g(\boldsymbol{\theta}) \geq 0 \\ \alpha_{2}-\left|\bar{\alpha}_{x}\right|+p g(\boldsymbol{\theta}) & \text { if } g(\boldsymbol{\theta})<0\end{cases}
$$

where

$$
g(\boldsymbol{\theta})=\frac{f_{1}}{f_{\min }}-1
$$

Two cases were tested: $n=6$ and $n=12$. In the former case, three feasible laminates yielded the minimum the longitudinal CTE of $\bar{\alpha}_{x}=8.5410^{-7} \mathrm{~K}^{-1}:\left[90_{2} / \pm 67.5 / 0_{8}\right]_{s}$, which had a first natural frequency of $f_{1}=156.25 \mathrm{~Hz},\left[90_{2} / 0_{2} / \pm 67.5 / 0_{6}\right]_{s}$, with $f_{1}=152.53 \mathrm{~Hz}$, and $\left[90_{2} / 0_{8} / \pm 67.5\right]_{s}$, with $f_{1}=151.36 \mathrm{~Hz}$ (these values are to be compared to the maximum frequency of the plate $f_{1}=183.71 \mathrm{~Hz}$ for $\left[90_{12}\right]_{s}$ and the minimum CTE for the unconstrained problem $\bar{\alpha}_{x}=-5.1710^{-8} \mathrm{~K}^{-1}$ for $\left.\left[ \pm 67.5 / \pm 45 / \pm 22.5_{3} / 0_{2}\right]_{s}\right)$. In the 
case $n=12$, the optimum laminate was $\left[90_{4} / \pm 67.5 / 0_{6} / \pm 22.5_{6}\right]$, with $\bar{\alpha}_{x}=5.9510^{-7} \mathrm{~K}^{-1}$ and $f_{1}=150.13$ $\mathrm{Hz}$.

For this problem, the penalty parameter was set to $p=1.010^{-4}$. The allowable fiber orientations, genetic operators, and other parameters were the same as for the in-plane problem. The bandwidth found by the maximum likelihood method were $\sigma=0.2$ for $n=6$ and $\sigma=0.15$ for $n=12$. The reliability of the standard GA, UMDA and DDOA is shown in Figure 5. The reliability displays the same trend as for the

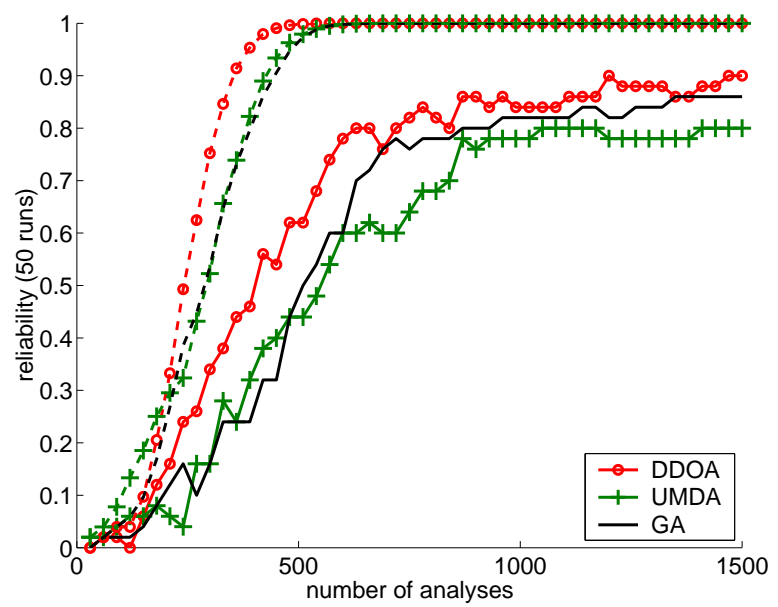

(a)

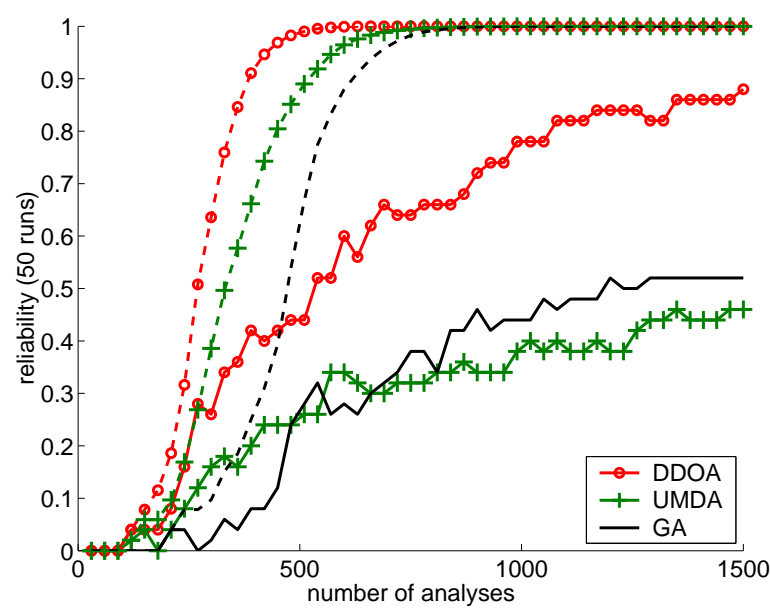

(b)

Figure 5. Reliability (solid line) and cumulative reliability (reliability of the algorithm when the best points visited are stored, dashed line) of a standard genetic algorithm, the univariate marginal distribution algorithm, and the double-distribution algorithm for the in-plane laminate optimization problem (6 variables (a) and 12 variables (b)).

in-plane problem: no significant difference can be observed in the efficiency of the GA and UMDA, but the superiority of DDOA appears clearly, even for a problem of such a moderate size. As for the in-plane problem, the benefit of accounting for variable dependencies through the lamination parameter distribution $f\left(V_{1}^{*}, V_{3}^{*}, W_{1}^{*}, W_{3}^{*}\right)$ increases with $n$.

\section{Discussion}

The maximum likelihood method constitutes a convenient way of determining a good value for the bandwidth $\sigma$. However, since the study is based on the first generation, it only provides an upper bound for $\sigma$ : because the distribution becomes more focused as the optimization progresses, the optimal bandwidth decreases with time. Experiments show that the best value for $\sigma$ is indeed smaller than those given by the maximum likelihood method. To further improve DDOA's efficiency, an adaptive strategy similar to that proposed by Bosnan and Thierens ${ }^{21}$ could be implemented.

A potential limitation of the proposed algorithm may be the lamination parameter-based acceptance procedure, which may become computationally expensive for large populations. The cost of calculating all target-candidate distances can be avoided by implementing a probabilistic acceptance procedure, instead of the current deterministic method. Alternatively, other cooperation schemes between the design variables and the auxiliary variables may be devised.

As far as absolute efficiency is concerned, standard techniques, such as an elitist strategy, or different selection methods, could be employed to improve reliability. However, these were not used in this study to allow a fair comparison between algorithms.

\section{Concluding Remarks}

A new approach for composite laminate optimization was proposed. The algorithm, called doubledistribution optimization algorithm, is based on a simple version of estimation of distribution algorithm, the univariate marginal distribution algorithm, but corrects the distribution inaccuracy caused by the inde- 
pendent variable assumption. The new idea of the method rests on the use of the distribution of integral variables, the lamination parameters, to incorporate some dependencies, which obviates the need for complex probabilistic models that can be expensive to construct and overly sensitive to sampling errors.

The method was demonstrated on two composite optimization problems, one in-plane problem, and one problem that combines extensional and flexural properties. In both cases, DDOA outperformed the basic UMDA, as well as a standard GA. In addition, the superiority of the double-distribution algorithm increased with the problem dimension. These results demonstrate the effectiveness of the approach for problems that can be expressed in terms of the lamination parameters. The case of problems that are not functions of the lamination parameters only (such as strength maximization problems) will be investigated in a future paper.

The authors would like to emphasize the fact that the double-distribution approach is not restricted to laminate optimization, but can be adapted to any problem that involves multiple-level variables, in particular all problems that introduce integral quantities, such as the moments of inertia in structural dynamics, the drag coefficient in aerodynamics, the permeability in the study of flows in porous media, etc. In all these situations, the distribution of higher-level quantities provides information about the lower-level variable dependencies and can be used to improve the efficiency of the search.

\section{References}

${ }^{1}$ Le Riche, R. and Haftka, R., "Optimization of Laminate Stacking Sequence for Buckling Load Maximization by Genetic Algorithm," AIAA Journal, Vol. 31, No. 5, 1993, pp. 951-957.

${ }^{2}$ Punch, W., Averill, R., Goodman, E., Lin, S.-C., and Ding, Y., "Using Genetic Algorithms to Design Laminated Composite Structures," IEEE Intelligent Systems, Vol. 10, No. 1, 1995, pp. 42-49.

${ }^{3}$ McMahon, M., Watson, L., Soremekun, G., Gürdal, Z., and Haftka, R., "A Fortran 90 Genetic Algorithm module for composite laminate structure design," Engineering with Computers, Vol. 14, No. 3, 1998, pp. 260-273.

${ }^{4}$ Larrañaga, P. and Lozano, J., Estimation of Distribution Algorithms, Kluwer Academic Publishers, 2001.

${ }^{5}$ Baluja, S., "Population-Based Incremental Learning: A Method for Integrating Genetic Search Based Function Optimization and Competitive Learning,," Tech. Rep. CMU-CS-94-163, Carnegie Mellon University, Pittsburgh, PA, 1994.

${ }^{6}$ Mühlenbein, H. and Mahnig, T., "Evolutionary Algorithms: From Recombination to Search Distributions," Theoretical Aspects of Evolutionary Computing, 2000, pp. 137-176.

${ }^{7}$ Mühlenbein, H. and Mahnig, T., "Evolutionary Algorithms and the Boltzmann Distribution," Proceedings of the Foundations of Genetic Algorithms VII conference, 2002.

${ }^{8}$ De Bonet, J., Isbell, Jr., C., and Viola, P., "MIMIC: Finding Optima by Estimating Probability Densities," Advances in Neural Information Processing Systems, edited by M. C. Mozer, M. I. Jordan, and T. Petsche, Vol. 9, The MIT Press, 1997, p. 424.

${ }^{9}$ Pelikan, M. and Mühlenbein, H., "The Bivariate Marginal Distribution Algorithm," Advances in Soft Computing Engineering Design and Manufacturing, edited by R. Roy, T. Furuhashi, and P. K. Chawdhry, Springer-Verlag, London, 1999, pp. 521-535.

${ }^{10}$ Mühlenbein, H. and Mahnig, T., "FDA-A scalable evolutionary algorithm for the optimization of additively decomposed functions," Evolutionary Computation, Vol. 7, No. 1, 1999, pp. 45-68.

${ }^{11}$ Pelikan, M., Goldberg, D., and Cantú-Paz, E., "BOA: The Bayesian Optimization Algorithm," Proceedings of the Genetic and Evolutionary Computation Conference GECCO-99, edited by W. Banzhaf, J. Daida, A. E. Eiben, M. H. Garzon, V. Honavar, M. Jakiela, and R. E. Smith, Vol. I, Morgan Kaufmann Publishers, San Fransisco, CA, Orlando, FL, 13-17 1999, pp. 525-532.

${ }^{12}$ Tsai, S. and Pagano, N., "Invariant Properties of Composite Materials," Composite Materials Workshop, edited by S. Tsai, J. Halpin, and N. Pagano, 1968.

${ }^{13}$ Miki, M., "Optimum Design of Laminated Composite Plates subject to axial Compression," Proc. Japan-U.S. CCM-III, edited by K. Kawabata and S. U. A. Kobayashi, 1986, pp. 673-680.

${ }^{14}$ Todoroki, A. and Haftka, R., "Lamination Parameters for Efficient Genetic Optimization of the Stacking Sequence of Composite Panels," Proc. $7^{\text {th }}$ AIAA/USAF/NASA/ISSMO Multidisciplinary Analysis and Optimization Symposium, 1998, pp. 870-879.

${ }^{15}$ Foldager, J., Hansen, J., and Olhoff, N., "A General Approach forcing Convexity of Ply Angle Optimization in Composite Laminates," Structural Optimization, Vol. 16, 1998, pp. 201-211.

${ }^{16}$ Gürdal, Z., Haftka, R., and Hajela, P., Design and Optimization of Laminated Composite Materials, John Wiley \& Sons, Inc., 1998.

${ }^{17}$ Sebag, M. and Ducoulombier, A., "Extending Population-Based Incremental Learning to Continuous Search Spaces," Proceedings of the 5th Conference on Parallel Problems Solving from Nature, edited by T. Bäck, G. Eiben, M. Schoenauer, and H.-P. Schwefel, Springer Verlag, 1998, pp. 418-427.

${ }^{18}$ Gallagher, M., Frean, M., and Downs, T., "Real-valued Evolutionary Optimization using a Flexible Probability Density Estimator," Proceedings of the Genetic and Evolutionary Computation Conference, edited by W. Banzhaf, J. Daida, A. E. Eiben, M. H. Garzon, V. Honavar, M. Jakiela, and R. E. Smith, Morgan Kaufmann, Orlando, Florida, USA, 1999 , pp. 840-846.

${ }^{19}$ Bosman, P. A. N. and Thierens, D., "Continuous iterated density estimation evolutionary algorithms within the IDEA framework," Optimization By Building and Using Probabilistic, Las Vegas, Nevada, USA, 2000, pp. 197-200. 
${ }^{20}$ Diaconu, C., Sato, M., and Sekine, H., "Feasible Region in general Design Space of Lamination Parameters of Laminated Composites," AIAA Journal, Vol. 50, No. 3, 2002, pp. 559-565.

${ }^{21}$ Bosman, P. and Thierens, D., "Expanding from discrete to continuous estimation of distribution algorithms: The IDEA," Proceedings of the Sixth International Conference on Parallel Problem Solving From Nature - PPSN VI, Springer-Verlag, 2000, pp. 767-776.

${ }^{22}$ Turlach, B., "Bandwidth selection in kernel density estimation: A review," Tech. Rep. 9317, C.O.R.E. and Institut de Statistique, Université Catholique de Louvain, 1993.

\section{A. Choice of a good bandwidth for the kernel density estimate}

The choice of the bandwidth $\sigma$ is essential for a good estimation of $f(\mathbf{V})$ and hence for the efficiency of DDOA. An upper bound of the optimal bandwidth can be found by obtaining the value of $\sigma$ that provides the best estimation of the distribution of selected points at the first generation.

A cross-validation method was used to choose the value of $\sigma$, as described by Turlach. ${ }^{22}$ The method is a modified maximum likelihood approach, where the likelihood is replaced by the pseudo-likelihood:

$$
L(\sigma)=\prod_{i=1}^{\mu} f_{\sigma, i}\left(\mathbf{V}_{i}\right),
$$

where $f_{\sigma, i}$ is a leave-one-out estimation of the density at $\mathbf{V}_{i}$, based on the $\mu-1$ other data points:

$$
f_{\sigma, i}=\sum_{\substack{j=1 \\ j \neq i}}^{\mu} K\left(\mathbf{V}-\mathbf{V}_{i}\right)
$$

The bandwidth $\sigma$ was allowed to take value from $\{0.04,0.05,0.06,0.07,0.08,0.09,0.1,0.12,0.15,0.2,0.3,0.4\}$. To reduce the variance of the likelihood estimation, the total likelihood $L^{\text {all }}$ over 100 independent runs was used as a measure of the goodness of the density estimation, for each value of $\sigma$ :

$$
L^{\text {all }}=\prod_{r=1}^{100} L_{r}(\sigma),
$$

where $L_{r}(\sigma)$ is the likelihood obtained for the $r^{\text {th }}$ run. Given the small sample size of $\mu=30$ and the sample creation procedure, the set of selected points is highly likely to contain duplicates of the same individual, hence compromising the validity of the cross-validation. Therefore, the contribution of $f_{\sigma, i}\left(\mathbf{V}_{i}\right)$ at points that had been used to construct the approximation was ignored. The procedure was applied for the two optimization problems treated in this paper: the constrained maximization of $A_{22}$ and the constrained minimization of the longitudinal CTE, for $n=6$ and $n=12$. The $\log$ likelihood as a function of $\sigma$ is shown in Figures 6 and 7. All curves display the same trend: a very low likelihood for small values of $\sigma(\sigma<0.1)$, followed by a relatively flat region. This indicates that when too small kernels are used, the predictive power of the approximation is compromised, while the quality of the estimation is not very sensitive to the choice of $\sigma$ beyond $\sigma=0.2$. For the two problems, the best bandwidth was $\sigma^{*}=0.2$ for $n=6$ and $\sigma^{*}=0.15$ for $n=12$.

\section{Acknowledgements}

This work was partly funded by AFOSR grant F49620-09-1-0070. 


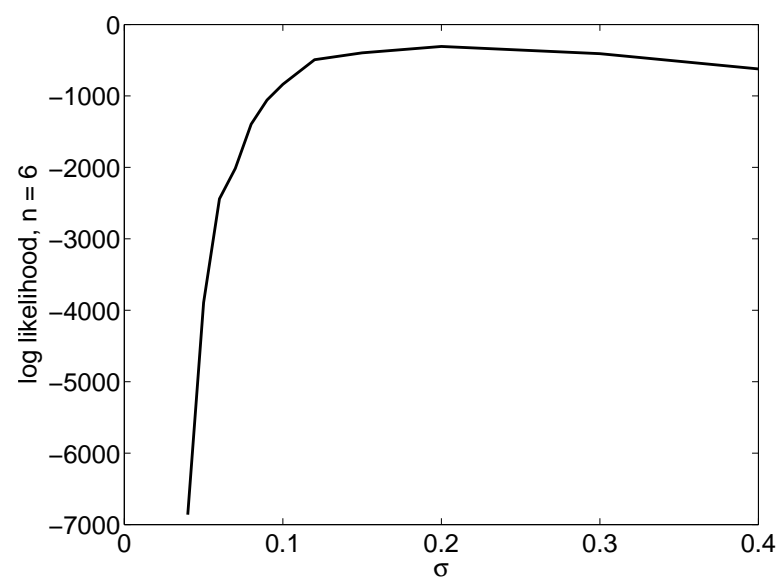

(a)

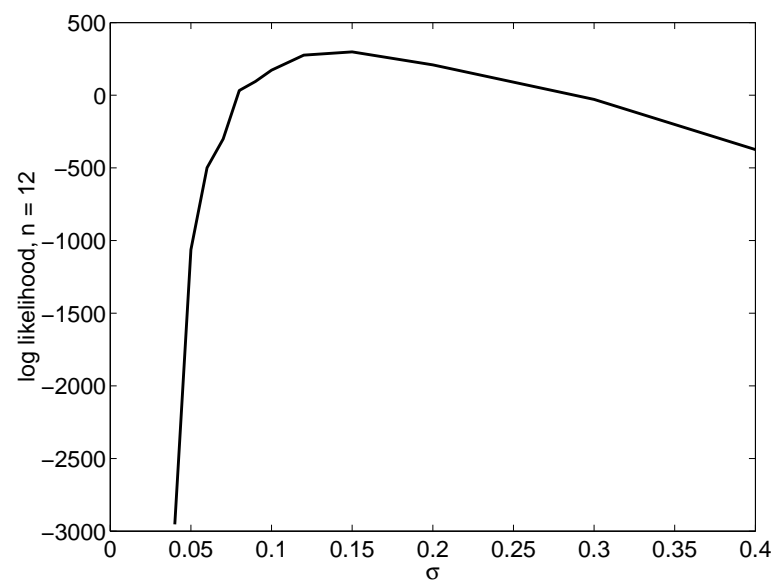

(b)

Figure 6. Log likelihood as a function of $\sigma$ for $n=6$ (a) and $n=12$ (b), $\mu=30$, in-plane problem

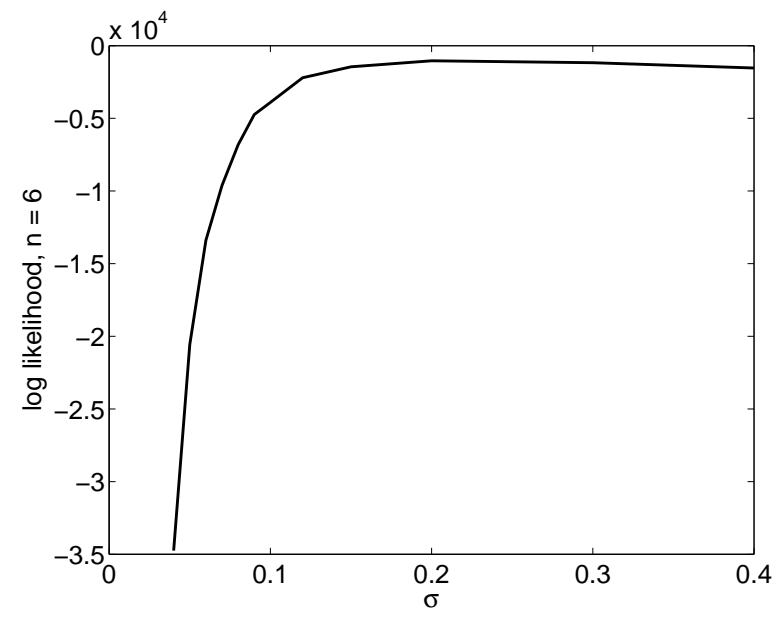

(a)

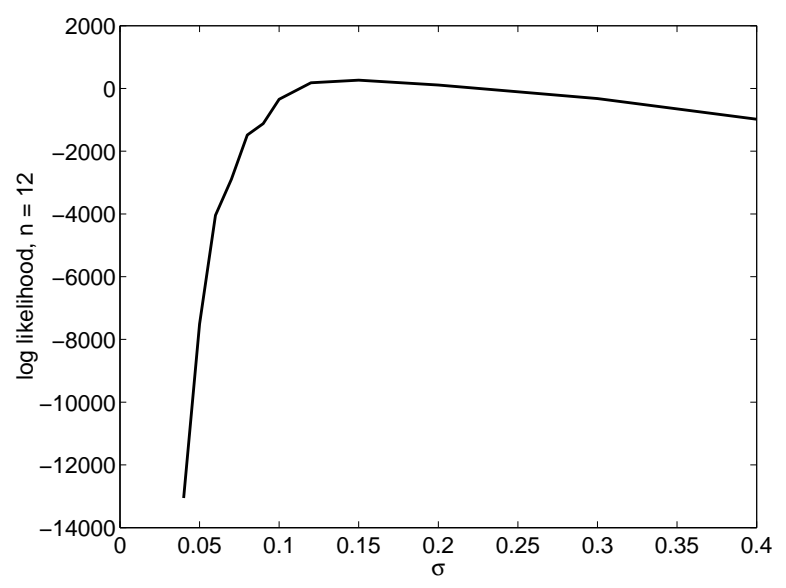

(b)

Figure 7. Log likelihood as a function of $\sigma$ for $n=6$ (a) and $n=12$ (b), $\mu=30$, out-of-plane problem 